development of clinical disease and associated changes in immune status, gut and energy homeostasis.

Results Animals fed a HFD showed lower autoantibody titres going along with an improved overall survival and a tendentiously lower infiltration of the kidney by leukocytes. Beneficial clinical effects were reflected in systemic immunologic changes, as the distribution and differentiation of main immune cell subsets in HFD animals more closely resembled that of yet healthy animals. We assume that most probably a complex interplay of different fiber-associated effects underlies these favorable effects. This may involve intestinal leakage and bacterial translocation that were increased in LFD animals. Further, LFD animals showed a significant increase in body weight and white adipose tissue expressing more leptin and inflammatory cytokines. We are currently testing, if the observed beneficial effects may also be attributed to an increased fermentation of dietary fibre into SCFA. SCFA intersect in various ways and at different sites with the immune system and mostly have anti-inflammatory effects.

Conclusion Altogether, we think that intake of dietary fiber affects immune status, gut and energy homeostasis. These may be interlinked and affect each other, inflicting more or less systemic chronic inflammation promoting lupus pathology.

Acknowledgment This work was supported by the B. Braun Stiftung, the Forschungskommission Freiburg and the Ministry of Science, Research, and Arts Baden-Wurttemberg (Margarete von Wrangell Programm).

\section{P56 RHUPUS SYNDROME IN A TERTIARY HOSPITAL}

Isabel Martínez-Cordellat, Roxana González-Mazarío, Marta De-la-Rubia-Navarro, Cristobal Pavez-Perales, Samuel Leal-Rodriguez, José Ivorra-Cortés, Inmaculada ChalmetaVerdejo, Elena Grau-García, Cristina Alcañiz-Escandell, Jorge Juan Fragio-Gil, Luis GonzálezPuig, Rosa Negueroles-Albuixech, José Eloy Oller-Rodríguez, Francisco Miguel Ortiz-Sanjuán, Elvira Vicens-Bernabeu, Carmen Nájera-Herranz, Inés Cánovas-Olmos, José Andrés RománIvorra. Rheumatology Dept., HUP La Fe, Valencia, Spain

\subsection{6/lupus-2020-eurolupus. 103}

Background/Purpose Rhupus syndrome (RhS) is a rare combination of Rheumatoid Arthritis (RA) and Systemic Lupus Erythematosus (SLE). Different studies describe RhS cases that begin with erosive arthritis and the presence of rheumatoid factor (RF) and/or anti CCP and then the SLE symptoms.

Despite the fact that RhS shows a low prevalence, it would be useful to know clinical characteristics of RhS patients since their therapy and outcome differ from those having RA or SLE alone.

Methods Retrospective study with systematic revision of electronic clinical records of RhS patients was performed. Demographic, clinical and immunological data were collected.

Results Eight RhS patients were included (all fulfilled SLICC 2012 criteria for SLE and ACR 2010 for RA). Mean age was 67.3 (45-84) years (7 were female).

In 3 cases RA was the first diagnosis with a mean evolution of 4.5 years until SLE diagnosis. In contrast, in 5 cases SLE was the first diagnosis with a mean evolution of 7.2 years until RA diagnosis. Photosensitivity and arthritis were the predominant clinical manifestations. One patient presents pericarditis and other case showed rheumatoid nodules in elbows. Renal, pulmonary or neurological affection was no reported.

4 patients were under biological/JAK inhibitors therapies (2 abatacept, 1 rituximab and 1 baricitinib) with favorable response of treatment.
Conclusions In contrast to other series, only the $37.5 \%$ of our RhS cases begins with polyarticular seropositive arthritis. The $62.5 \%$ started with SLE symptoms as haematological alterations, cutaneous and serological manifestation, and showed longer progression to have polyarticular affection. Thus, RhS diagnosis is earlier in patients that begin with RA symptoms. $4 \mathrm{RhS}$ patients were refractory to DMARd treatments, where biological/JAK inhibitors therapies are needed.

\section{P57 SMOKING AND PRIMARY CHRONIC CUTANEOUS LUPUS: WHO ARE THE MOST VULNERABLE?}

${ }^{1}$ Cristina Drenkard, ${ }^{2}$ Laura Aspey, ${ }^{3}$ Charles Helmick, ${ }^{1}$ Gaobin Bao, ${ }^{1} S$ Sam Lim. ${ }^{1}$ Division of Rheumatology, Emory University, Atlanta; ${ }^{2}$ Dept. of Dermatology, Emory University, Atlanta; ${ }^{3}$ Centers for Disease Control and Prevention, Division of Population Health, Atlanta, USA

\subsection{6/lupus-2020-eurolupus.104}

Background/Purpose Chronic Cutaneous Lupus Erythematosus (CCLE), including discoid lupus, often leads to scarring and disproportionately afflicts African American (AA) people. Smoking worsens the severity of skin lupus and is highly prevalent in those from disadvantaged groups. We examined sociodemographic disparities in tobacco smoking among patients with CCLE confined to the skin (primary CCLE [pCCLE]).

Methods Cross-sectional study of adults with dermatologistdiagnosed pCCLE consented into the Georgians Organized Against Lupus (GOAL) Cohort. GOAL is a population-based lupus cohort established in the Southeastern US, where there is a large AA, socioeconomically disadvantaged population. pCCLE were classified as never smokers (NS, <100 lifetime cigarettes), former smokers (FS, $\geq 100$ lifetime cigarettes and not currently smoking), and current smokers (CS, $\geq 100$ lifetime cigarettes and currently smoking). We created a Disadvantage Score (DScore) by attributing 1 point to each of the following: living below the federal poverty level, $\leq$ high school education, self-reported AA race, unemployed/disabled, self-perceived discrimination, and moderate/severe depression. We examined the association of DScore with active smoking (CS vs NS) and smoking cessation (CS vs FS).

Results Among 124 patients (86\% females, 82\% AA), the prevalence of NS, FS, and CS was 53\%, 16\%, and $31 \%$, respectively. In multivariate models adjusting for age, sex and dermatology visits (table 1), the odds of CS (vs NS) increased significantly as the DScores increased $(\mathrm{OR}=3.9$ and

Abstract P57 Table 1 Association of tobacco smoking status with disadvantage score among adults with Primary CCLE. Multivariate Analysis*

\begin{tabular}{lllll}
\hline $\begin{array}{l}\text { Disadvantage } \\
\text { score }\end{array}$ & $\begin{array}{l}\text { CS vs NS } \\
\text { OR }(95 \% \mathrm{Cl})\end{array}$ & P-value & $\begin{array}{l}\text { CS vs FS } \\
\text { OR }(95 \% \mathrm{Cl})\end{array}$ & P-value \\
\hline $0-1$ & $($ Ref) & (Ref) & (Ref) & (Ref) \\
$2-3$ & $3.9(1.1-13.3)$ & 0.03 & $6.9(1.5-31.7)$ & 0.01 \\
$4-6$ & $9.3(2.5-34.6)$ & 0.003 & $7.6(1.6-35.6)$ & 0.004 \\
\hline
\end{tabular}

*Multivariate logistic regression adjusted for significant confounders (age, gender, and dermatology visits). ^ Disadvantage score represents the sum of 1 point for each of the following characteristics: living below the federal poverty level, $\leq$ high school, African American race, unemployed/disabled, self-perceived discrimination, moderate to severe depressive symptoms.

Abbreviations: NS=Never Smoker; FS=Former Smoker; $\mathrm{CS}=$ Current Smoker; OR=Odds Ratio; $\mathrm{Cl}=$ Confidence Interval; ref=Reference Group. 
$\mathrm{OR}=9.3$ for adults with DScores of 2-3 and 4-6 [compared with DS 0-1], respectively). Odds of CS (vs FS) were also higher with higher DScores $(\mathrm{OR}=6.9$ and $\mathrm{OR}=7.6$ for adults with a DS of 2-3 and 4-6 [compared with DS 0-1], respectively).

Conclusion Smoking is highly prevalent in patients with pCCLE. DScores were positively associated with CS and inversely associated with FS. Smoking cessation is particularly important for adults with pCCLE, and such efforts should target individuals from the most disadvantaged sociodemographic groups.

Acknowledgements The GOAL Cohort is supported by the Centers for Disease Control and Prevention (CDC) Grant 1U01DP005119. The content of this research is solely the responsibility of the authors and does not necessarily represent the official views of the CDC. The authors have no conflicts of interest to declare.

\section{P58 SYSTEMIC LUPUS ERYTHEMATOSUS IN NATIVE SUB- SAHARAN AFRICANS: A SYSTEMATIC REVIEW AND META-ANALYSIS}

\begin{abstract}
${ }^{1}$ Mickael Essouma, ${ }^{1} J a n$ René Nkeck, ${ }^{2}$ Francky Teddy A Endomba, ${ }^{3,4}$ Jean Joel Bigna, 1,5 Madeleine Singwe-Ngandeu, ${ }^{6}$ Eric Hachulla. ${ }^{1}$ Faculty of Medicine and Biomedical Sciences, University of Yaoundé I, Yaoundé, Cameroon; ${ }^{2}$ University of Bourgogne, Dijon, France; ${ }^{3}$ Centre Pasteur of Cameroon, Yaoundé, Cameroon; ${ }^{4}$ Faculty of Medicine, University of Paris Sud XI, Le Kremlin-Bicêtre, France; ${ }^{5}$ Yaoundé Central Hospital, Yaoundé, Cameroon;

${ }^{6}$ Claude Huriez Hospital, Lille University, Lille, France
\end{abstract}

\subsection{6/lupus-2020-eurolupus. 105}

Background This systematic review of literature and meta-analysis aimed to determine the prevalence, phenotype and treatment of systemic lupus erythematosus (SLE) in Native subSaharan Africans.

Methods PubMed, EMBASE, Web of Science, African Journals Online, and Global Index Medicus as well as references of retrieved papers were searched to select studies addressing SLE in Native sub-Saharan Africans and published during January 1, 2008- October 7, 2018. Results were pooled through narrative review and random-effects model. Heterogeneity $\left(\mathrm{I}^{2}\right)$ was assessed via the $\chi^{2}$ test. Pooled estimates are expressed with 95\% confidence intervals. This study is registered with PROSPERO: registration number CRD42019139226.

Results Fifteen hospital-based studies were included out of 1502 records. The pooled prevalence of SLE was $1.7 \%(0.8-$ 2.9). The mean age at diagnosis ranged from 28.8 to 39.2 years. The female proportion was $88 \%-100 \%$. Rheumatological (5.1\%-99.9\%), dermatological (4.3\%-100\%) and hematological (1.4-86.9\%) manifestations were the commonest. Patients had a high seroprevalence for anti-ribonucleoprotein 57.9\% (36.4-77.9), anti-Smith 53.5\% (40.4-66.2), anti-Sjogren syndrome antigen A $45.6 \%(19.2-73.4)$ and anti-Sjogren syndrome antigen B 33.7\% (13.6-57.6) autoantibodies. The most used treatments were corticosteroids 99\% (94.9-100) and antimalarials 62. 8\% (23.3-94.1). The pooled mortality rate was $10.3 \%$ (3.3-20.6); mainly due to infections, kidney and neurological involvement.

Conclusions Over the last 30 years, SLE was not rare among Native sub-Saharan Africans and its featured characteristics were earlier onset, female predominance, and high seropositivity for extractable nuclear antigen autoantibodies. The standard treatments were corticosteroids and antimalarials. The mortality rate was high. Population prevalence and incidence as well as full description of SLE characteristics in Native subSaharan Africans are needed.

\section{P59 PREDICTORS OF RENAL SURVIVAL IN A COHORT OF PATIENTS WITH LUPUS NEPHRITIS WITH MORE THAN 30 YEARS OF FOLLOW-UP}

${ }^{1}$ Filipa Farinha, ${ }^{2}$ Ruth J Pepper, ${ }^{3}$ Daniel G Oliveira, ${ }^{1}$ Thomas McDonnell, 'David A Isenberg, ${ }^{1}$ Anisur Rahman. ${ }^{1}$ Centre for Rheumatology, University College London, London; ${ }^{2}$ Centre for Nephrology, University College London-Royal Free Campus, London, UK; ${ }^{3}$ Internal Medicine Dept., Centro Hospitalar e Universitário do Porto, Porto, Portugal

\subsection{6/lupus-2020-eurolupus. 106}

Background Despite the improvement in survival of patients with lupus nephritis ( $\mathrm{LN}$ ) over the last decades, LN is associated with progression to end-stage renal disease (ESRD) in a significant proportion of patients. We aimed to investigate the factors influencing renal survival in patients with LN.
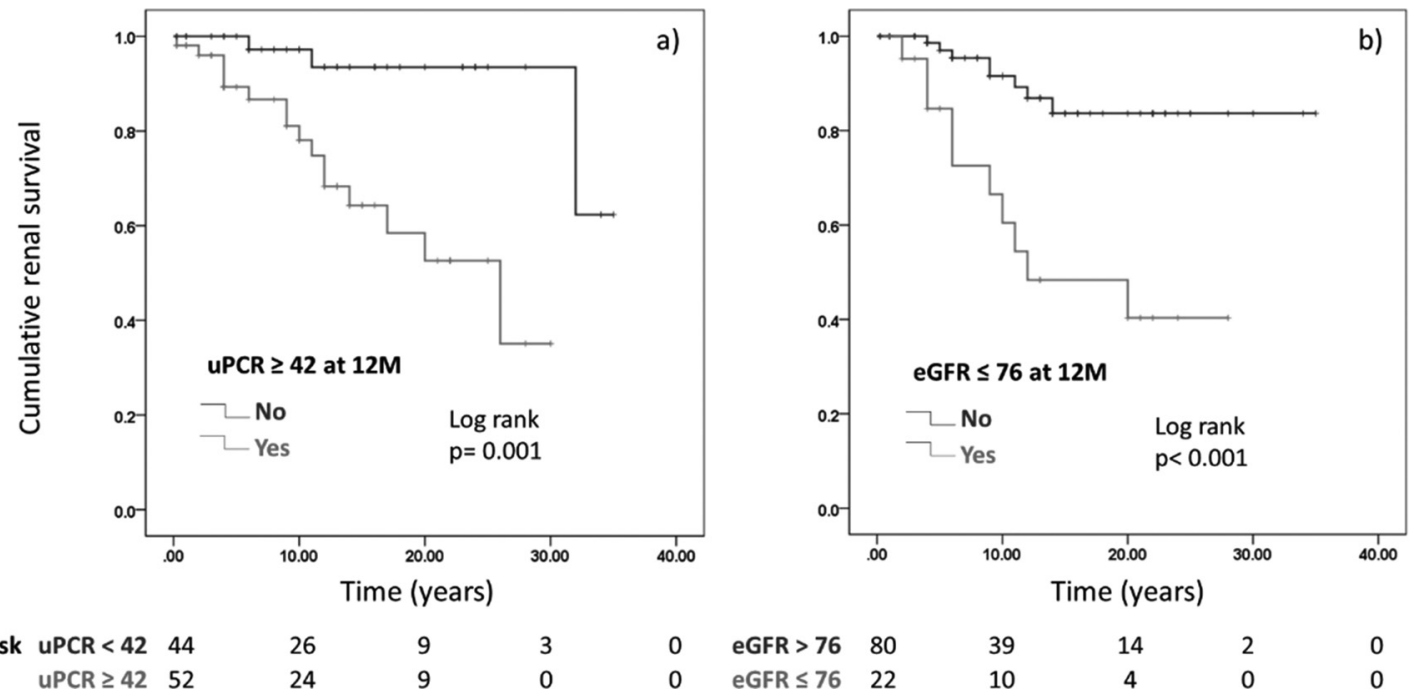

Abstract P59 Figure 1 Kaplan-meier curves showing cumulative renal survival for patients with LN 